\title{
T cell ALL in a child with Ataxia telangiectasia; diagnosis and management challenge
}

\author{
Omaima Ahmed ${ }^{1}$, Yara Felimban ${ }^{2}$, and Abeer Almehdar ${ }^{1}$ \\ ${ }^{1}$ King Abdulaziz Medical City - Jeddah \\ ${ }^{2}$ Maternity and Children Hospital
}

November 23, 2020

\begin{abstract}
Ataxia telangiectasia (A-T) is a rare childhood autosomal recessive neurodegenerative chromosomal instability disorder. It is characterized by high risk of hematological malignancies with T-cell phenotype being the most common, which can present first before the diagnosis of A-T made. The chromosomal instability in A-T increases the toxicity to radio- chemotherapeutic agents, creating the treatment modification challenges and the deviation from the optimal management protocols. In this case report we present a 14-month-old boy diagnosed as T cell -ALL. Based on his early presentation, family history of childhood lymphoma, and high AFP, inherited predisposition was suspected, and genetic testing confirm A-T. This report represents the crucial part of clinical suspicion of A-T in similar cases as well as highlighting the importance of an early A-T diagnosis that prevents toxic death due to the extensive regimen of radio- chemotherapeutic agents. The report summarizes the toxicity and modification challenges during management with literature review for the chemotherapy modification experience in such cases.
\end{abstract}

T cell ALL in a child with Ataxia telangiectasia; diagnosis and management challenges Ahmed Omaima ${ }^{1 *}$, Felimban Yara $\mathbf{S}^{2}$, Al-Mehdar Abeer ${ }^{3}$

${ }^{1}$ Pediatric Hematology Oncology department, Princess Nora Oncology center, King Abdul-Aziz Medical city, Jeddah, Saudi Arabia

${ }^{2}$ Pediatric department, Maternity and Children Hospital, Dammam, Saudi Arabia

${ }^{3}$ Radiology department, King Abdul-Aziz Medical city, Jeddah, Saudi Arabia

*Correspondence to:

Omaima S Ahmed, MD, Pediatric Hematology Oncology department, Princess Nora Oncology center, King Abdul-Aziz Medical city, Jeddah, Saudi Arabia, PO box 9515, Jeddah 24123, Tel: 00966-503610887, Email: e_omayma@hotmail.com

Text Word count: 2380 words

Abstract Word count: 159 words

Brief running title: T cell ALL in a child with Ataxia telangiectasia

Key words: T cell ALL, Ataxia Telangiectasia, Chemotherapy modification

Number of manuscript pages: 7 pages

Number of Tables: 3 tables

Number of Figures: 4 figures 
Abbreviations

\begin{tabular}{ll}
\hline AT & Ataxia Telangiectasia \\
ALL & Acute lymphoblastic leukemia \\
ATM & ataxia telangiectasia mutated gene \\
AFP & Serum alpha-fetoprotein \\
SVC & Superior Vena cava \\
CALL08 & Childhood Acute lymphoblastic leukemia protocol 2018 ( local protocol) \\
MRD & Minimal residual disease \\
DI & delayed intensification \\
MTX & Methotrexate \\
CTCAE & Common Terminology Criteria for Adverse Events \\
UMN & Upper Motor Neuron \\
GCS & Glasgow Coma Scale \\
HC & hemorrhagic cystitis \\
NHL & Non-Hodgkin's Lymphoma \\
HD & Hodgkin's Lymphoma \\
CR & Complete Remission \\
EFS & Event Free survival \\
\hline
\end{tabular}

Case Report

T cell ALL in a child with Ataxia telangiectasia: diagnosis and management challenges

\section{Ahmed Omaima, Felimban Yara S, Al-Mehdar Abeer}

Abstract: Ataxia telangiectasia (A-T) is a rare childhood autosomal recessive neurodegenerative chromosomal instability disorder. It is characterized by high risk of hematological malignancies with T-cell phenotype being the most common, which can present first before the diagnosis of $\mathrm{A}-\mathrm{T}$ made. The chromosomal instability in A-T increases the toxicity to radio- chemotherapeutic agents, creating the treatment modification challenges and the deviation from the optimal management protocols. In this case report we present a 14-month-old boy diagnosed as T cell -ALL. Based on his early presentation, family history of childhood lymphoma, and high AFP, inherited predisposition was suspected, and genetic testing confirm A-T. This report represents the crucial part of clinical suspicion of A-T in similar cases as well as highlighting the importance of an early A-T diagnosis that prevents toxic death due to the extensive regimen of radio- chemotherapeutic agents. The report summarizes the toxicity and modification challenges during management with literature review for the chemotherapy modification experience in such cases.

\section{BACKGROUND}

Ataxia telangiectasia (A-T) is a complex, multisystem, rare autosomal recessive disorder caused by mutations in the ATM (ataxia telangiectasia mutated) gene on chromosome 11q.26 (1). Patients usually present before the age of three years with progressive cerebellar ataxia; however, other neurological manifestation including ocular apraxia and dysarthria and oculocutaneous telangiectasia developed few years later. Almost $50 \%$ of patients with A-T have variable degrees of immune deficiency and can present with recurrent Sinopulmonary infection. In comparison to general population, patients with A-T have 100 folds that increase risk of developing hematological malignancies, mainly aggressive Non-Hodgkin lymphoma, acute leukemia, and Hodgkin lymphoma. Among acute leukemia, T-cell is the commonest phenotype. Some patients may present with cancer before the diagnosis of A-T is made. Early diagnosis might be suspected if there is positive family history, early onset malignancy, and an unusual severe toxicity after chemotherapeutic treatment (2). Serum alpha-fetoprotein is a simple, rapid, and reliable screening test for A-T; it is proven to be elevated in more than $90 \%$ of A-T cases (3). Increasing toxicity in A-T patients with acute leukemia after radio- and chemotherapeutic treatment remains a subject of debate. (2) 
In this paper, we discuss the value of AFP in raising the suspicions of the disease, and the importance of the early diagnosis of $\mathrm{A}-\mathrm{T}$ which can prevent the patient from toxic death as a result of radio- chemotherapy toxicity. After analyzing the modification and outcome in each chemotherapy phase, we found that despite the modification of the patient's chemotherapy, he had an early and rapid neurological toxicity in his last chemotherapy phase.

We conclude that the optimal treatment of malignancy in A-T patient is not well defined due to the variation in phenotypes and clinical presentations; therefore, it is difficult to standardize the therapy. Appropriate modification of the therapy should be individualized and continuously reviewed at each stage without disregarding several factors as age at presentation, clinical condition, and the immune deficiency status associated with A-T. Among published cases, we found no evidence of a preferred strategy as each has its cons and pros. As we seek to elucidate the optimal treatment strategy in future, we are optimistic that reporting similar case presentations, diagnostic strategies, chemotherapy modifications, toxicity, and toxic death in consequence may be of great benefit

\section{CASE PRESENTATION}

We are presenting a 14 months old boy who was born to a healthy consanguine parent. He is a product of full term, uncomplicated pregnancy and delivery. His eldest sister is healthy; however, his older brother died of sepsis at the age of 18 months shortly after being diagnosed with lymphoblastic lymphoma at our hospital.

Our patient was transferred to our PICU after an acute onset of shortness of breath and cyanosis as case of mediastinal mass for diagnosis and management. Upon admission, he had a picture of superior vena cava syndrome and hepato-splenomegaly; however, there was no clinically palpable lymph node. CNS and testicular exam were unremarkable. The initial labs showed leukocytosis $(>70 x 109 / \mathrm{L})$, elevated LDH, elevated uric acid, mildly low immunoglobulin (IgG) level and high alpha-feto protein (table1). He was intubated on day 2 of admission and mechanically ventilated for 16 days. The initial Chest X-ray showed enlarged mediastinum with subtotal opacification in the right hemi-thorax along with bilateral pleural effusion (Fig 1a). Chest CT revealed a mediastinal mass compressing on the lower part of trachea (Fig 2a). The peripheral blood sample fLowcytometry was consistent with T-cell Lymphoblastic Leukemia/ Lymphoma. There was 16\% cell expressing CD1a, CD2, cyCD3(bright), CD4, CD5, CD7, CD8(partial/dim), CD45 \& CD123; negative for smCD3, CD10, CD13, CD16, CD19, CD33, CD34, CD44, CD56, CD57, CD99, CD117, TDT \& HLADR. Cytogenetic was 46XY, $+7, \operatorname{der}(7 ; 7)(7 \mathrm{pter} 7 \mathrm{p} 15:: 7 \mathrm{q} 3 ? 47 \mathrm{p} 11.2:: 7 \mathrm{p} 11.27 \mathrm{q} 3 ? 4:: 7 \mathrm{p} 157 \mathrm{pter})[10]$ and 46,idem,?del(6)(pterq21:)[5]/46,XY[5] . It revealed a male chromosome complement with two related abnormal cell lines. The (stem line) showed a dicenteric derivative chromosome 7 with possible uncertain inversion. The (sideline) showed the stem line abnormality complement with uncertain deletion within the long arm of chromosome 6. FISH confirmed Isodicentric chromosome 7q. ECHO showed small ASD secondum, multiple apical VSD, and compressed SVC with mild pericardial effusion, but normal left cardiac function. On further evaluation, he was found to have central hypothyroidism MRI showed normal pituitary with normal pituitary area on a cerebellar atrophy (Fig $3 \mathrm{a} \& \mathrm{~b}$ ); therefore, he was started on levothyroxine by endocrine team.

Early diagnosis was made because of his early presentation as he presented with low $\operatorname{IgG}$ and had positive family history of childhood lymphoma. Genetics and Immunology teams were involved. Underlying immunodeficiency was suspected; therefore, the patient was planned for 4weeks IVIG. Whole exome sequence was sent.

\section{Management course}

Patient started immediate chemotherapy as per local protocol CALL08 High risk Arm (C) (4). He completed unmodified induction and augmented consolidation phase. He achieved hematological and radiological remission post induction. Bone marrow flowcytometry (FC) on day 15 and 29, confirmed negative (MRD less than 0.01\%). CXR and CT chest at the end of induction confirmed resolved mediastinal mass (Fig 1b \& $2 \mathrm{~b})$. 
By end of augmented consolidation phase, the whole exome sequence was consistent with the diagnosis of A-T; it detected homozygous variant in ATM gene, c.9066del (p. Gly3023Alafs*10); Chr11 (GRCh37): g.108236130del and both parents were heterozygous. Genetics counseling was done.

The patient's diagnostic radiological exposure was minimized during his management course; his chemotherapy was downgraded to Arm B (4) and modified as shown in (Table 2\&3). The Interim Maintenance I (High dose Methotrexate) phase was replaced by Capizzi; he received Single Capizzi and delayed intensification (DI) in which Doxorubicin was reduced by $20 \%$. Nevertheless, we planned to reduce cyclophosphamide by $50 \%$ and to add Mesna. As we were concerned of the increasing risk of hemorrhagic cystitis due to recurrent hematuria, cyclophosphamide was omitted in DI phase. During (IM) Capizzi, he tolerated the 3 escalating MTX doses (100-150-200 mg/m2) but didn't tolerate the 4th dose $(250 \mathrm{mg} / \mathrm{m} 2)$. He developed grade III mucositis and prolonged fever neutropenia. Therefore, the 5th dose was omitted. Our patient completed 18/36 months on maintenance chemotherapy receiving Vincristine monthly and Intrathecal Methotrexate every 3 months. His daily oral Mercaptopurine and weekly oral Methotrexate started as $60 \%$ of total dose, and then was gradually upgraded reaching $100 \%$ of optimal dose.

His chemotherapy was stopped at month 18 of Maintenance due to acute neurological progression likely related methotrexate and vincristine. The patient remained ventilator dependent with persistent slow improving GCS until his death one year after therapy discontinuation.

\section{Comorbidities and toxicity}

As shown in (Table 3), the patient developed CTCAE grade III on top of cytopenia and infection during his chemotherapy, in addition to Peg Asperginase related severe hypertriglyceridemia. His triglyceride level reached $22.9 \mathrm{mmol} / \mathrm{L}$ (Normal value $0.50-2.23 \mathrm{mmol} / \mathrm{L}$ ). It resolved spontaneously on low fat diet. By the end of the induction phase, he developed peripheral neuropathy as a result of Vincristine toxicity; it was resolved by the mid of the consolidation after a course of pyridostigmine and pyridoxine. Only during Cappizi and DI Modification was needed, when at the end of the 4th cycle of Capizzi, he had mucositis GI grade III with prolonged fever neutropenia. Therefore, his 5th Capizzi cycle was omitted. On DI phase, he developed recurrent hematuria with prolonged thrombocytopenia, in which he was admitted for clinical typhlitis and was managed conservatively.

During Maintenance phase, he was admitted 3 times due to mild to moderate chest infection and asthma exacerbations that resolved with IV antibiotics and steroids. At month 18 of Maintenance, we stopped his chemotherapy treatment because of acute neurological progression that happened 3 month after his Intrathecal Methotrexate and one month after Vincristine dose, as he was on full oral Methotrexate dose as well.

Initially, the patient was presented with decreased activity and progressive hypotonia (head lag, unable to sit, and UMN facial palsy with aphasia). His CSF was negative for relapse and infection which showed normal glucose and protein. EEG showed no epileptic discharges. However, brain MRI reported changes that were likely due to post chemotherapy effect; Persistent diffuse brain volume loss with prominent ventricular system and enlargement CSF space, bilateral mild white matter periventricular at the level of the occipital horn (Fig 4 a \& b).

The patient then developed progressive decrease level of consciousness (GCS 9 -12) and desaturated with acidosis; therefore, he required mechanical ventilation. After a multidisciplinary case conference, we decided to stop chemotherapy and keep him on supportive care management. Despite chemotherapy modification and close monitoring for myelotoxicity and the risk of secondary malignancy, he had an early and sudden rapid neuro-toxicity, most likely related to methotrexate and vincristine, which made him remain ventilator dependent with persistent low GCS 10, he remain ventilator dependent with slow improving GCS to12 until his death one Year after therapy discontinuation.

\section{Discussion}

Ataxia telangiectasia is a rare, autosomal dominant, inherited, and progressive neurodegenerative disease. 
It is characterized by immunodeficiency, sensitivity to ionizing radiation, genomic instability, and increased predisposition to cancer, especially to lymphoid malignancies (6). Its incidence of malignancy is $24.5 \%$ in the largest cohort (7). Malignancy can present early and can precede the diagnosis of A-T. Our patient and his brother were both presented with a very early onset of $\mathrm{T}$ cell neoplasm (less than 2 years) even before the symptoms of A-T appeared.

Serum alpha-fetoprotein is a simple, rapid, and diagnostic screening test for A-T; it is proven to be elevated (levels above $30 \mathrm{ng}$ ) in more than $90 \%$ of A-T cases. AFP level test was done for our patient in investigation for mediastinal mass. The result was high as it was high for his older brother as well. Our patient showed no typical symptoms of A-T. However, elevated levels of AFP raised our suspicions to make the diagnosis of A-T highly likely. Further investigations of genetic testing confirmed our diagnosis.

Chromosome abnormality involving chromosomes 7 and 14 are typically identified in individuals with A-T. Translocations with break points are commonly at 14q11 (the T-cell receptor-alpha/delta locus) and at 14q32 (immunoglobulin heavy chain gene), 7p14 7q35 resulting in immunoglobulin/T-cell receptor gene trans- or cross-rearrangements. Other abnormality such as inversion of chromosome 7 or 14 was also reported (12). In our case, the cytogenetic showed (stem line) of dicentric derivative chromosome 7 with possible inversion. FISH confirmed Isodicentric chromosome 7q.

Due to lack of evidence-based protocol to follow, chemotherapy modification strategy remains datable, depending on the center's experience in such cases. Many reports disclosed excessive and fatal toxicity as a result of radiotherapy treatment; therefore, there is a consensus to avoid craniospinal radiation therapy (11).

As reported by Cohen et al, cyclophosphamide has shown to have serious side effects including toxic hemorrhagic cystitis (HC) and life-threatening bleeding due to bladder wall telangiectasia in A-T patients. It is usually severe with Cyclophosphamide dose above $1200 \mathrm{mg} / \mathrm{m}^{2}$ (9). Our patient developed grade II HC after the first dose of Cyclophosphamide at a dose of $1 \mathrm{gm} / \mathrm{m} 2$ and received no further dosages.

Some case reports advice to modify neurotoxic drugs (such as Vincristine) because A-T patients have decreased ability to compensate muscle weakness (13). It may be safer if vincristine is discontinued, delivered at a reduced dosage, or replaced by Vinblastine whenever early signs of muscle weakness are noticed. Our patient developed bilateral foot drop as a result of VCR toxicity which was managed with a course of pyridostigmine and pyridoxine. Maximum dose of Vincristine was then resumed after complete recovery with no further symptoms' recurrence.

A-T patients are prone to methotrexate induced gastrointestinal tract toxicity; therefore, it is of great benefit to optimize drug clearance methods. As for our patient, we elected to cancel High dose Methotrexate and replace it with one CAPIZZI. The dose of MTX was adjusted according to tolerance. There was no use of folinic acid rescue for IV or OT MTX.

Multiple case series have described the results of chemotherapy treatment of lymphoid malignancies in patients with A-T. In 1980, Sandoval reported a cohort of A- T patients who developed lymphoid malignancies (NHL in 64\%, ALL in 15\%, and HD in 13\%). All patients receiving chemotherapy achieved complete remission with a survival rate 6-46 months. One patient died due to progressive disease after two months of treatment using prednisolone as monotherapy. Patients who received standard dose of chemotherapeutic treatment have shown better outcomes than modified induction (8). In our reported case, the patient received unmodified induction and consolidation due to the delay in the diagnosis of A-T. Although our patient did not develop sudden unusual severe toxicity, we elected to modify his chemotherapy based on most reported cases in the literature review as described above.

In addition to cytotoxic drugs, A-T patients experience radiotherapy treatment toxicity as well. In a systemic review by Schoenaker, $23 \mathrm{~A}-\mathrm{T}$ cases were conducted and analyzed in which all achieved complete remission except one. Both modified and unmodified treatment strategies can induce complete remission; there's no significant difference in achieving CR (94\%). However, unmodified treatment is better tolerated in younger A-T patients than in older A-T patients. Unmodified treatment shows higher survival rates than modified 
treatment, $73 \%$ and $57 \%$ respectively. Upfront modified treatment events were related to toxicity and not caused by relapse. Modified treatment may lead lower EFS; yet, it has less toxicity and better life quality. Apart from regular immunoglobulin replacement therapy, no additional supportive care is needed in A-T patients (2). Our Patient achieved CR after unmodified induction and maintained it with the chemotherapy modification. Apart from the acute neuro-toxicity, all other symptoms were manageable and reversible.

In conclusion, A-T should be suspected in any child who presents early with T cell ALL, family history, high AFP, and low immunoglobulins. Treatment of ALL in children with A-T is feasible as long as the diagnosis is made early. To prevent the child from toxic death and secondary malignancy due to unnecessary exposure to radio- and chemotherapeutic agents, early family genetic counseling is of great value. Nevertheless, unmodified induction treatment strategies are successful to induce complete remission. Treatment can be modified according to the patient's clinical condition, immune deficiency status, beside tolerance, and type of toxicity developed. Despite the modification of chemotherapy and the close monitoring for myelotoxicity, the patient may develop acute methotrexate and vincristine related neuro-toxicity. Neuro-toxicity can be avoided by either folinic acid rescue or modification of the Vincristine vs Vinblastine dosage prophylactically. Further evidence-based medicine is strongly needed to elucidate the optimal treatment strategy.

\section{REFERENCES}

1. P. Malcolm Taylor, Ataxia-telangiectasia in children, Guidance on diagnosis and clinical care, Nottingham University Hospitals. The Ataxia-Telangiectasia Society 2014

2. T. Szczepanski, N. Mahlaoui, J.L. Loeffen, Treatment of acute leukemia in children with ataxia telangiectasia / M.H.D. Schoenaker et al./European Journal of Medical Genetics 59 (2016) 641e646

3. Thomas a. waldmann k. robert mcintire, serum-alpha-fetoprotein levelsin patients with ataxiatelangiectasia Metabolism Branch and Laboratory of Cell Biology,National Cancer Institute, Bethesda, Maryland 20014, U.S.A., the lancet Nov 25, 1972

4. Jastaniah W, Elimam N, Abdalla K, AlAzmi AA, Elgaml AM, Alkassar A, Daghistani M, Felimban S Early vs. late MRD response- and risk-based treatment intensification of childhood acute lymphoblastic leukemia: a prospective pilot study from Saudi Arabia.Exp Hematol Oncol. 2018 Nov 19; 7:29. doi: 10.1186/s40164-018-0121-x. e Collection 2018.PMID:30479872

5. T O Crawford, R L Skolasky, R Fernandez, K J Rosquist, H M Lederman, Survival probability in ataxia telangiectasia 2006 Archives of Disease in Childhood

6. Taylor AM, Metcalfe JA, Thick J, Leukemia and lymphoma in ataxia telangiectasia, Blood 1996 $87: 423-438$

7. Suarez, F., Mahlaoui, N., Canioni, D., Andria manga, C., et al., 2015. Incidence, presentation, and prognosis of malignancies in ataxia-telangiectasia: a report from the French national registry of primary immune deficiencies. J. Clin. Oncol. 33 -2, 202e208, 10.

8. Sandoval, C., Swift, M., 1998 Dec. Treatment of lymphoid malignancies in patients with ataxiatelangiectasia. Med. Pediatr. Oncol. 31 (6), 491e497

9. Cohen, J.M., Cuckow, P., Davies, E.G., 2008 May. Bladder wall telangiectasia causing life-threatening hematuria in ataxia-telangiectasia: a new observation. ActaPaediatr. 97 (5), 667e669

10. Ussowicz, M., Musia ł, J., Duszenko, E., Haus, O., et al., 2013. Long-term survival after allogeneicmatched sibling PBSC transplantation with conditioning consisting of low-dose busilvex and fludarabine in a 3-year-old boy with ataxia telangiectasia syndrome and ALL. Bone Marrow Transplant. 48 (5), $740 \mathrm{e} 741$.

11. Yanofsky, R.A., Seshia, S.S., Dawson, A.J., Stobart, K., et al., 2009. Ataxia-telangiectasia: atypical presentation and toxicity of cancer treatment. Can. J. Neurol. Sci.36 (4), 462e467

12. Gatti, R., 1993-2016. Ataxia-telangiectasia. In: Pagon, R.A., Adam, M.P., Ardinger, H.H., Wallace, S.E., Amemiya, A., Bean, L.J.H., Bird, T.D., Dolan, C.R.,Fong, C.T., Smith, R.J.H., Stephens, K. (Eds.), GeneReviews@ [Internet]. University of Washington, Seattle, Seattle (WA).

13. Barbara pietrucha1, edyta heropolitañska-pliszka1, richard a. gatti2, ewa bernatowska, AtaxiaTelangiectasia: guidelines for diagnosis and comprehensive care, The David Geffen School of Medicine at UCLA, Los Angeles, USA, 2007 
Table (1) Initial blood result

Table (2) Chemotherapy modification

Table (3) Grade 3 and above CTCAE Chemotherapy toxicity and modification

Fig (1a\&b) CXR Pre and post Induction

Frontal chest x-ray images (a) at the time of diagnosis and (b) post induction. Large mediastinal opacity (star) is identified in the right Para tracheal of the superior mediastinum. Note also the right side pleural effusion (arrow). Resolution of the mediastinal mass on the chest x-ray post treatment.

Fig ( 2 a\& b) CT chest Pre and post Induction

Selected axial images from the CT scan of the chest. (a)Trans-axial image from the non-enhanced CT scan of the chest at the time of diagnosis demonstrates the large right mediastinal soft tissue mass at the level of the heart. (b) trans-axial image from the contrast enhanced CT scan of the chest after the induction demonstrate the mediastinal mass with significant reduction in size.

Fig (3 a \&b) MRI brain Base line

Selected images from the MRI of the brain before the chemotherapy treatment. (a) Sagittal T1 weighted MR image at the midline demonstrate the mild cerebellar atrophy (arrow). (b, c) selected axial T2 weighted images of the supratentorial brain shows no evidence of white matter change

Fig (4 a \& b). MRI brain Post neuro-toxicity

Selected axial FLAIR MR images (a, b) of the brain post chemotherapy demonstrate the development of the periventricular white matter changes (arrows) suggestive of the toxicity.

\section{Hosted file}

Table 1 initial blood work.pdf available at https://authorea.com/users/377876/articles/ 494525-t-cell-all-in-a-child-with-ataxia-telangiectasia-diagnosis-and-managementchallenge

\section{Hosted file}

Table 2 chemotherapy modifications.pdf available at https://authorea.com/users/377876/ articles/494525-t-cell-all-in-a-child-with-ataxia-telangiectasia-diagnosis-and-

management-challenge

\section{Hosted file}

Table 3 toxicity Grading.pdf available at https://authorea.com/users/377876/articles/494525t-cell-all-in-a-child-with-ataxia-telangiectasia-diagnosis-and-management-challenge 

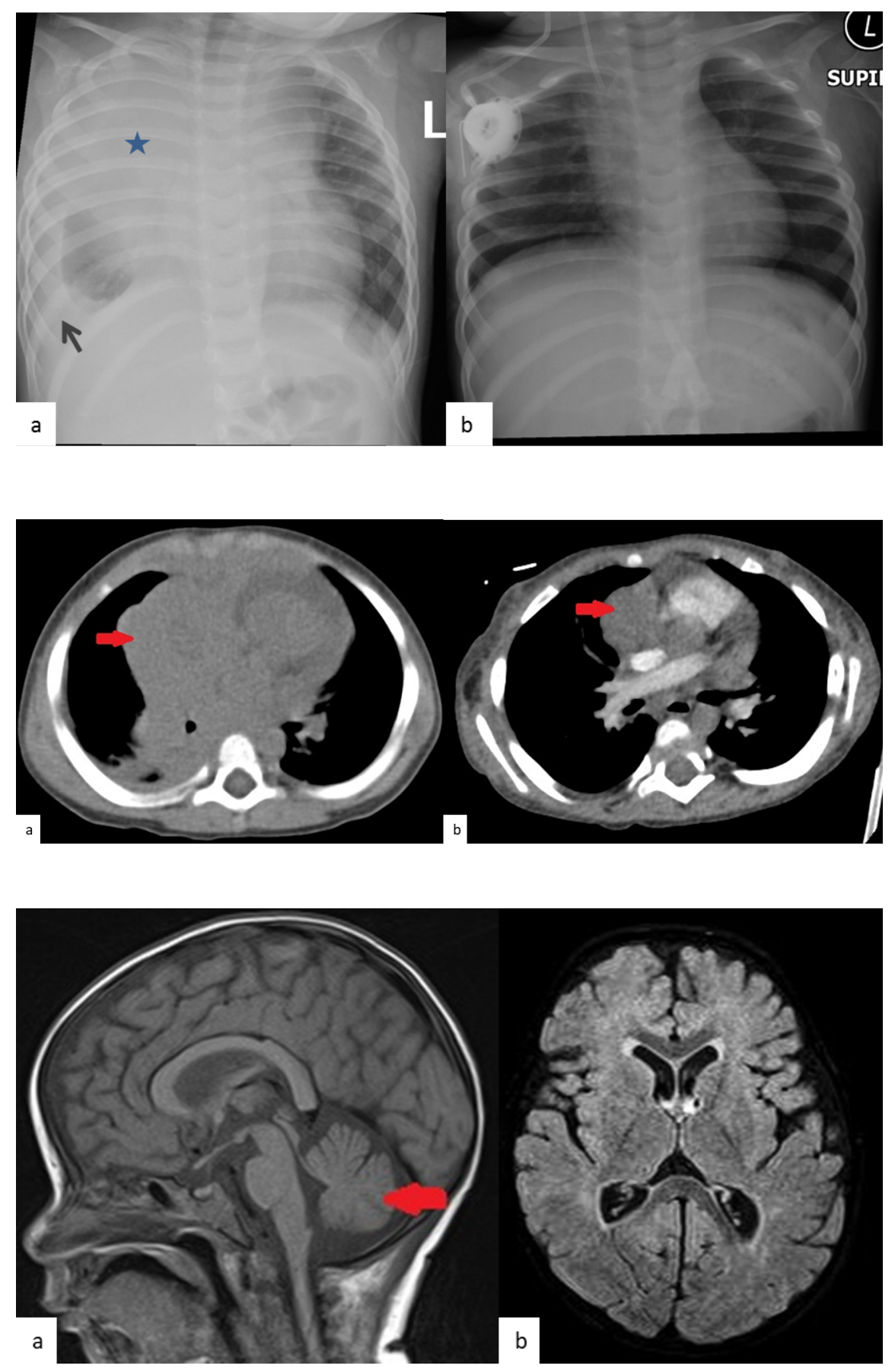


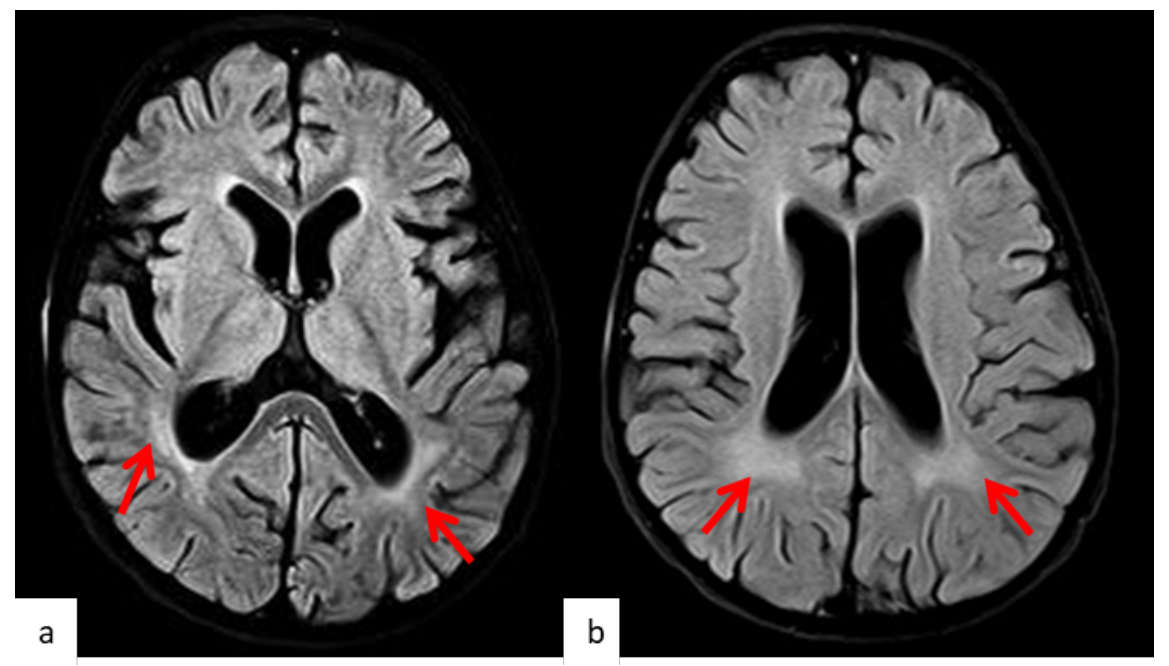

